\title{
A!
}

This is an electronic reprint of the original article.

This reprint may differ from the original in pagination and typographic detail.

Vercruyssen, N.; Verhagen, T.G.A.; Flokstra, M.G.; Pekola, J.P.; Klapwijk, T.M.

\section{Evanescent states and nonequilibrium in driven superconducting nanowires}

Published in:

Physical Review B

DOI:

10.1103/PhysRevB.85.224503

Published: 04/06/2012

Document Version

Publisher's PDF, also known as Version of record

Please cite the original version:

Vercruyssen, N., Verhagen, T. G. A., Flokstra, M. G., Pekola, J. P., \& Klapwijk, T. M. (2012). Evanescent states and nonequilibrium in driven superconducting nanowires. Physical Review B, 85(22), 1-10. [224503].

https://doi.org/10.1103/PhysRevB.85.224503

This material is protected by copyright and other intellectual property rights, and duplication or sale of all or part of any of the repository collections is not permitted, except that material may be duplicated by you for your research use or educational purposes in electronic or print form. You must obtain permission for any other use. Electronic or print copies may not be offered, whether for sale or otherwise to anyone who is not an authorised user. 


\title{
Evanescent states and nonequilibrium in driven superconducting nanowires
}

\author{
N. Vercruyssen, ${ }^{1}$ T. G. A. Verhagen, ${ }^{1, *}$ M. G. Flokstra, ${ }^{2}$ J. P. Pekola, ${ }^{3}$ and T. M. Klapwijk ${ }^{1}$ \\ ${ }^{1}$ Kavli Institute of Nanoscience, Delft University of Technology, 2628CJ Delft, The Netherlands \\ ${ }^{2}$ Kamerlingh Onnes Laboratory, Leiden Institute of Physics, P.O. Box 9504, 2300 RA Leiden, The Netherlands \\ ${ }^{3}$ O. V. Lounasmaa/Low Temperature Laboratory, Aalto University, P.O. Box 13500, FI-00076 AALTO, Finland
}

(Received 12 February 2012; published 4 June 2012)

\begin{abstract}
We study the nonlinear response of current transport in a superconducting diffusive nanowire between normal reservoirs. We demonstrate theoretically and experimentally the existence of two different superconducting states appearing when the wire is driven out of equilibrium by an applied bias, called the global and bimodal superconducting states. The different states are identified by using two-probe measurements of the wire, and measurements of the local density of states with tunneling probes. The analysis is performed within the framework of the quasiclassical kinetic equations for diffusive superconductors.
\end{abstract}

DOI: 10.1103/PhysRevB.85.224503

PACS number(s): 74.40.Gh, 74.78.Na, 74.25.fg, 74.45.+c

\section{INTRODUCTION}

Superconducting nanowires are often part of objects to study the Josephson effects in graphene, carbon nanotubes, or semiconducting nanowires. In addition, in many cases superconducting nanowires themselves are used to study their response to radiation. In most cases, the electron backscattering resistance is assumed to be located at the interfaces and in the normal metal part. An interesting question is to what extent the superconducting mesoscopic or (nano)wires themselves contribute to the resistance of a device due to the conversion from normal current to supercurrent and vice versa. For superconducting nanowires between superconducting contacts, a common assumption is that the applied power leads to dissipation and to an increased temperature varying over the wire length. ${ }^{1}$ In quite a few experiments with a nanowire between normal or superconducting pads, a parabolic temperature profile $T(x)$ is assumed to control the local superconducting properties. ${ }^{2-4}$ The definition of a temperature, however, requires that the electrons are in local equilibrium, a condition not easily met for wires of mesoscopic length scales. In the case of a biased normal wire, ${ }^{5}$ the diffusion time $\tau_{D}=L^{2} / D$, with $L$ the wire length and $D$ the diffusion constant, can be much shorter than the inelastic relaxation time $\tau_{\text {in }}$. In this case, the electron distribution is highly nonthermal and given by a two-step function $f(E, x)=(1-x) f_{0}(E-e V / 2)+x f_{0}(E+e V / 2)$, with $f_{0}(E, T)=1 /[\exp (E / k T)+1]$ a Fermi-Dirac distribution, $V$ the applied bias, $k$ Boltzmann's constant, $E$ the energy of the electrons measured from the Fermi energy, $T$ the bath temperature, and $x$ the coordinate along the wire. A general, nonthermal (or nonequilibrium) electron distribution in a superconductor influences almost all aspects of that superconductor. It affects the local Cooper-pair density and the current-carrying capacity, but it can also produce a voltage drop in the superconductor, i.e., a dc resistance of the superconductor. To discuss the various contributions, it is advantageous to separate the nonequilibrium distribution function $f(E)$ into an energy (or longitudinal) mode $f_{L}$, acting primarily on the amplitude of the superconducting gap, and a charge (or transverse) mode $f_{T}$, which leads to a shift in the pair chemical potential $\mu_{c p}{ }^{6}{ }^{6}$ The latter mode

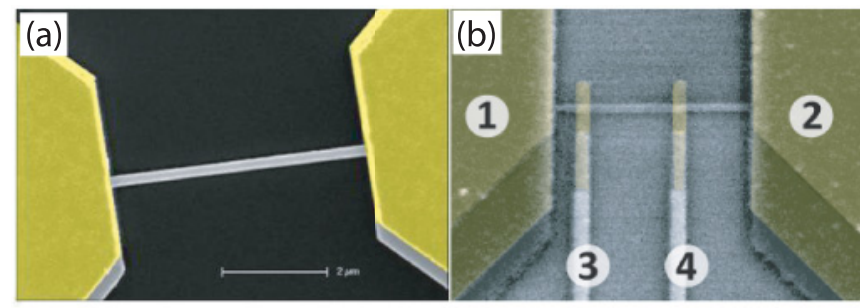

(c)

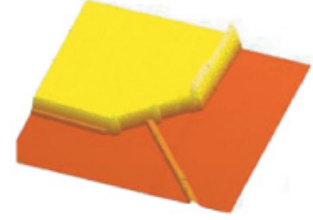

(d)

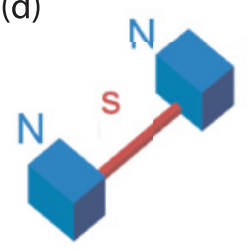

(e)

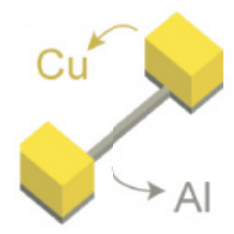

FIG. 1. (Color online) A superconducting Al nanowire connected to two massive normal reservoirs, consisting of the same Al, covered by a normal metal $\mathrm{Cu}$ layer: (a) scanning electron microscopy (SEM) picture, (c) atomic force microscope (AFM) picture, and (d), (e) schematic representation. The thin $\mathrm{Al}$ of the pads is driven normal by the inverse proximity effect of the thick normal $\mathrm{Cu}$. Normal tunneling probes are attached for local measurements (b).

$f_{T}$ describes an imbalance between electron and holes in the excitation spectrum, leading to a net charge $Q^{*}$ in the (decaying) excitations. This contribution can be dominant in experiments probing electrical transport in superconducting heterostructures at subgap energies.

In this paper, we report on an experimental and theoretical study of nonlinear electrical transport in a well-defined model system, ${ }^{7,8}$ in which a superconducting wire is connected to two large normal contact pads (Fig. 1). The normal electrodes induce evanescent subgap states in the superconducting wire. In addition, they act as equilibrium electron reservoirs to fill and empty the states in the superconducting wire. When a bias $e V$ is applied, evanescent electrons and holes are injected from the reservoirs into the superconducting wire, and the resulting nonequilibrium distribution function consists of both an energy mode $f_{L}$ and a charge mode $f_{T}$. The well-defined boundary conditions and simplicity of this system make it a natural 
(a) One Global Superconducting State

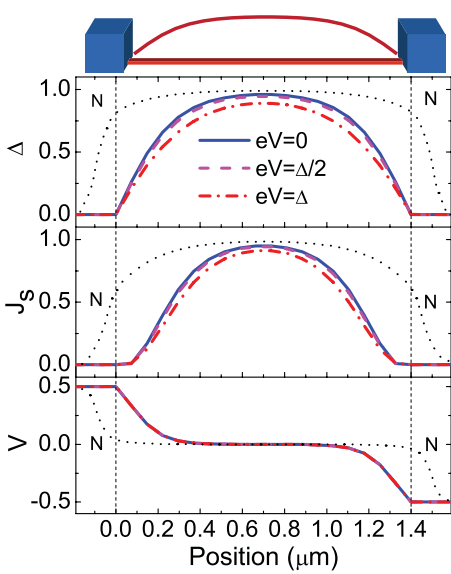

(b)

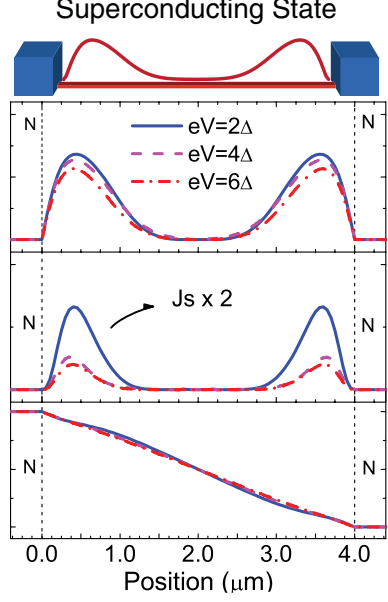

FIG. 2. (Color online) (a) The complete wire is in a single superconducting state with order parameter $\Delta(x)$. However, near the normal reservoirs, the condensate carries only a small fraction $J_{s}$ of the current as a supercurrent, which results in a resistance and a voltage drop at the ends of the wire, over roughly a coherence length. At the lowest temperatures, a small proximity effect can occur at the connection of the bilayer reservoirs to the wire (schematically illustrated by dotted black lines). (b) Two distinct superconducting domains at the ends of the wire are separated by a normal region in the center of the wire. Due to the small supercurrent, the voltage profile is almost equal to the normal state.

choice to study the superconducting state in the presence of a general nonequilibrium.

We address these microscopic properties of the wire experimentally using two-point measurements of the nanowire, which are a sensitive probe for the resistive properties originating in $f_{T}$. Measurements with tunneling probes allow us to measure the local density of states and the different chemical potentials involved. We demonstrate that two distinct metastable superconducting states exist when the wire is driven (Fig. 2). The first superconducting state extends over the complete length of the wire, and has been reported in the linear regime by Boogaard et al. ${ }^{9}$ The second state exists only under driving, and consists of two, geometrically separated, superconducting domains, both at the ends of the wire. We show that the superconductivity nucleates in the vicinity of the normal reservoirs because the local electron distribution is closer to the equilibrium state. The existence of metastable states has been identified in previous work using phenomenological models, ${ }^{10,11}$ based on a normal resistive domain. We analyze these states using the quasiclassical Green's functions, and show how the energy mode controls the existence of these states, whereas the charge mode controls the resistance. Hence, the full nonlinear response is found to be the result of a complex interplay between both the charge and the energy mode nonequilibrium.

\section{THEORETICAL FRAMEWORK}

We consider a model system consisting of a superconducting one-dimensional diffusive wire connected to two normal, equilibrium reservoirs [Fig. 1(d)]. Electrons are injected into

and extracted from the superconducting wire by the reservoirs with equilibrium Fermi distributions $f_{0}(E \pm e V, T)$, with relative Fermi levels determined by the applied voltage $V$. Within the wire, the electrons are distributed over the energies with a position- and energy-dependent nonequilibrium distribution function $f(E, x)$ determined by a diffusion equation. In addition, the electronic states concerned are decaying states, evanescent modes, as their energy is smaller than the energy gap $(e V \leqslant 2 \Delta)$. Therefore, it is necessary to include the interplay between these short-lived states and the superconducting condensate, which goes beyond a two-fluid description, in which a sharp distinction between long-living quasiparticle states and the condensate is assumed. Such an analysis is performed using the quasiclassical Green's functions theory for superconductivity, which treats the electronic properties of the excitations and the condensate on the same footing: ${ }^{12}$

$$
\check{G}=\left(\begin{array}{cc}
\hat{G}^{R} & \hat{G}^{K} \\
0 & \hat{G}^{A}
\end{array}\right), \quad \hat{G}^{R}=\left(\begin{array}{cc}
G & F_{1} \\
F_{2} & G^{\dagger}
\end{array}\right) .
$$

The retarded (advanced) functions $\hat{G}^{R(A)}$ consist of normal and anomalous propagators $G$ and $F$, which describe the single-electron spectrum and the coherence between electrons, respectively. The occupation numbers of the electronic excitations are contained in the Keldysh component $\hat{G}^{K}$.

In general, these Green's functions are dependent on the time, energy, position, and momentum of the particle: $G=$ $G(E, t, r, p)$. However, typical variations occur on a much slower length scale than the Fermi wavelength. The Green's functions are sharply peaked around the Fermi momentum $p=p_{F}$, and a considerable simplification can be obtained by integrating $G$ over all momenta. A second simplification arises from the short mean-free path in dirty superconducting films, which averages out any dependence on the momentum direction. The resulting equations were obtained by Usadel, ${ }^{13}$ and they only contain what is called the quasiclassical Green's functions $g(E, x, t)$ and $f(E, x, t)$. Our experimental observations indicate that relevant solutions are stationary, so in addition we neglect all time dependencies in the equations. This choice is partially supported by theoretical work of Snyman et al. ${ }^{14}$ who demonstrate for a simplified system that the solutions for a dc bias are always stationary. To parametrize $g(E, x)$ and $f(E, x)$, we use a complex pairing angle $\theta(E, x)$ describing correlation between electrons and holes, and a complex phase $\chi: g=\cos (\theta), f_{1,2}=\sin (\theta) e^{ \pm i \chi} .{ }^{15}$ The normalization condition $g^{2}+f_{1}^{\dagger} f_{2}=1$ is automatically fulfilled, while the variations of $\theta(E, x)$ and $\chi(E, x)$ are determined by the following diffusion equations:

$$
\begin{aligned}
& \hbar D\left\{\nabla^{2} \theta-\sin \theta \cos \theta(\nabla \chi)^{2}\right\} \\
& \quad=-i 2 E \sin \theta-\cos \theta\left(\Delta e^{-i \chi}+\Delta^{*} e^{i \chi}\right), \\
& \hbar D \nabla\left\{\sin ^{2} \theta(\nabla \chi)\right\}=i \sin \theta\left(\Delta e^{-i \chi}-\Delta^{*} e^{i \chi}\right),
\end{aligned}
$$

with $D$ the normal-state diffusion constant. The first equation describes how the presence of a local superconducting order parameter $\Delta(x)$ generates pair correlations $\theta(E, x)$, which allows us to calculate the local density of states (DOS) $N(E, x)=\operatorname{Re} \cos (\theta)$. The second equation relates the phase gradient of the gap to the presence of supercurrents. 
A convenient description of a nonequilibrium superconductor is obtained by introducing a generalized distribution function $h(E)$, defined as $G^{K}=G^{R} h(E)-h(E) G^{A}$. To disentangle the influence of the distribution function on the amplitude and the phase of the order parameter, $h(E)$ is split in the even part (energy mode) in particle-hole space $f_{L}(E, x)$, and the odd part (charge mode) $f_{T}(E, x)$. The total electron distribution functions $f(E, x)$ are then obtained from $2 f(E, x)=$ $1-f_{L}(E, x)-f_{T}(E, x)$. The presence of a charge mode is related to the presence of a charge $Q^{*}$ integrated over all excitations, and the consequence of inhomogeneity in the superconducting system, leading to conversion of quasiparticle current to supercurrent. Charge imbalance has been studied thoroughly at temperatures close to $T_{c}$, i.e., for long-lived quasiparticle excitations. ${ }^{16,17}$ However, the concept of charge imbalance also applies to short-lived evanescent states, ${ }^{18}$ for small injection voltages, and at low temperatures. ${ }^{19,20}$

Conservation of energy $E$ and charge $Q$ result in two coupled diffusion equations for $f_{L}$ and $f_{T}$ :

$$
\hbar D \nabla J_{E}=0, \hbar D \nabla J_{Q}=2 R_{L} f_{L}+2 R_{T} f_{T},
$$

with

$$
\begin{aligned}
J_{E} & =\Pi_{L} \nabla f_{L}+\Pi_{X} \nabla f_{T}+j_{\epsilon} f_{T}, \\
J_{Q} & =\Pi_{T} \nabla f_{T}-\Pi_{X} \nabla f_{L}+j_{\epsilon} f_{L} \\
\Pi_{L, T} & =1+|\cos \theta|^{2} \mp|\sin \theta|^{2} \cosh \left(2 \chi_{2}\right), \\
\Pi_{X} & =-|\sin \theta|^{2} \sinh \left(2 \chi_{2}\right), \quad j_{\epsilon}=2 \operatorname{Im}\left(\sin ^{2} \theta \nabla \chi\right), \\
R_{L, T} & =\operatorname{Re}\left[\sin \theta\left(\Delta e^{-i \chi} \mp \Delta^{*} e^{i \chi}\right)\right],
\end{aligned}
$$

where $\Pi_{L, T, X}$ are generalized diffusion constants, $j_{\epsilon}$ is the spectral supercurrent, and $R_{L, T}$ determine the magnitude of the source term on the right-hand side of Eq. (3). The energy current is dominated by the diffusion of the energy mode $f_{L}$. Our Al wires are relatively short, which means we can neglect inelastic processes, as the inelastic electron-electron and electron-phonon interaction lengths are of the order of $10 \mu \mathrm{m}$ at a temperature of $1 \mathrm{~K}^{21}$ For long wires or materials with a strong electron-phonon interaction, this is not necessarily true. The stronger electron-phonon coupling of $\mathrm{Nb}$ results in an inelastic mean-free path of roughly $0.1 \mu \mathrm{m} .^{22}$ The charge current consists partly of a normal current driven by a gradient of the charge mode $I_{n}=\Pi_{T} \nabla f_{T}$, and partly of a supercurrent related to a gradient of the phase $I_{s}=f_{L} j_{\epsilon}$. Conversion of a normal current into a supercurrent implies a change of $\nabla f_{T}$, and is proportional to $R_{T} \approx \Delta$ in Eq. (3).

The position-dependent potential in the superconductor $e \phi(x)$ is obtained by integrating the charge of the quasiparticle excitations over all energies:

$$
e \phi(x)=\int_{-\infty}^{\infty} N(E) f_{T}^{S}(E, x) .
$$

In order to conserve charge neutrality, the presence of the net charge in the excitations is compensated by a shift in the pair chemical potential $\delta \mu_{c p}(x)$. This means that the static electric field $E=\nabla \phi$, which drives the normal current $I_{n}$, does not influence the condensate since it is exactly balanced by $\delta \mu_{c p}(x)=-e \phi(x)$. If this were not the case, the Cooper pairs would accelerate.
The retarded and kinetic equations (2) and (3) are completed with the self-consistency relation for $\Delta(x)$ :

$$
\begin{aligned}
\Delta(x)= & \frac{N_{0} V_{\mathrm{eff}}}{4 i} \int_{-\hbar \omega_{D}}^{\hbar \omega_{D}} d E\left(\sin \theta e^{i \chi}-\sin \theta^{*} e^{i \chi^{*}}\right) f_{L} \\
& -\left(\sin \theta e^{i \chi}-\sin \theta^{*} e^{i \chi^{*}}\right) f_{T} .
\end{aligned}
$$

The charge mode is directly related to the observed potential drop over the superconductor through Eq. (5), and the energy mode $f_{L}$ only appears implicitly in the gap Eq. (6).

\section{POSSIBLE SOLUTIONS}

In this section, we present the numerical solutions of Eqs. (2)-(6) for the model system shown in Fig. 1. The wire can be considered to be one dimensional, as the width and thickness are smaller than the dirty superconducting coherence length $w, t \leqslant \xi=\sqrt{\frac{\hbar D}{2 \Delta_{0}}}$. The normal equilibrium reservoirs act as boundary conditions, both for the superconducting pairing angles $\theta=\nabla \chi=0$ and the distribution functions $f_{L, T}$. Temperature enters the problem only through the boundary conditions for $f_{L}$ and $f_{T}$, while all nonequilibrium processes in the wire itself are contained in the distribution functions. After an initial guess for $\Delta(x)$, the superconducting angles $\theta$ and $\chi$ are calculated from the retarded equations (2). Subsequently, the kinetic equations (3) can be solved to obtain $f_{L}$ and $f_{T}$. Finally, the value of $\Delta(x)$ is updated using Eq. (6), and this process is repeated until all values converge. We find two distinct superconducting solutions for the problem: (a) one global superconducting state [Fig. 2(a)] and (b) a bimodal superconducting state separated by a normal valley [Fig. 2(b)].

\section{A. One global superconducting state}

The first solution is characterized by one coherent superconducting state, which extends over the full length of the wire, although the strength of the superconducting gap $\Delta$ is suppressed at the edge of the wire by the presence of the normal reservoirs [Fig. 2(a)]. Although fully superconducting, the wire has a finite resistance due to the conversion of a normal current into a supercurrent, as shown by the position-dependent voltage $V$. Normal electrons, which are injected from the metallic reservoirs, decay into Cooper pairs over roughly one coherence length $\xi$. The excess charge $Q^{*}$ associated with the charge mode $f_{T}$ of these evanescent quasiparticle states results in the presence of an electric field in the superconductor, and hence a potential drop over the same length the supercurrent increases. These processes correlate with the picture of electrons being injected at energies $E \approx e V$, leading to a two-step distribution $f_{L}$, as shown previously by Keizer et al. ${ }^{7}$ While the charge mode nonequilibrium $f_{T}$ relaxes over a length scale of $\xi$, because of interaction with the condensate, the energy mode $f_{L}$ remains constant over the length of the wire due to the absence of inelastic interactions [Fig. 3(a)].

For increasing voltages, there is hardly any change in the profiles of $\Delta, \phi, J_{s, n}, f_{L, T}$ until the wire switches to the normal state. For example, there is no gradual expansion of the voltage-carrying parts at the end of the wires, as one would guess qualitatively. A careful analysis ${ }^{7}$ indicates that the energy mode $f_{L}$ triggers this transition, while the current is still far below the critical pair-breaking current $I_{c 0}$. 
(a) One Global Superconducting State
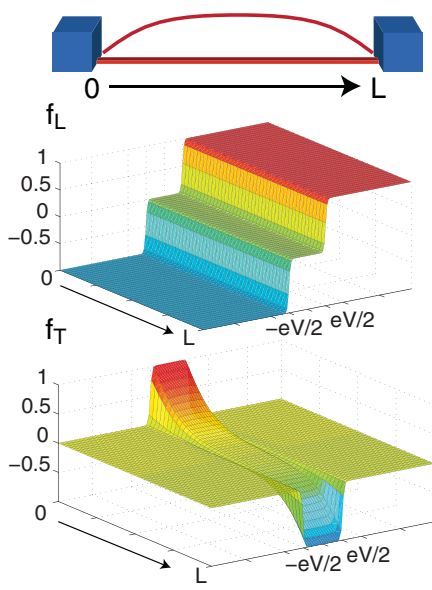

(b)

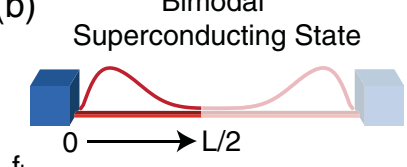

$f_{L}$
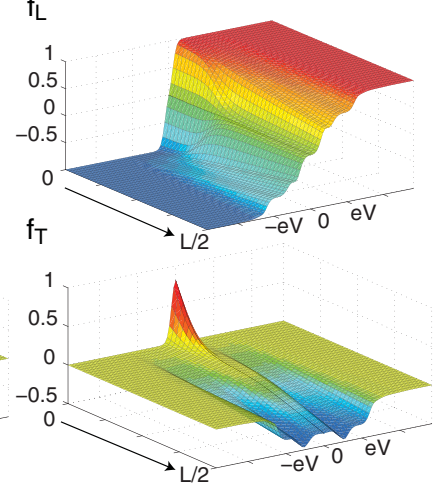

FIG. 3. (Color online) The even mode $f_{L}$ and odd mode $f_{T}$ of the nonequilibrium distribution function $f(E, x)$. (a) For the global superconducting state, a two-step distribution is present through the full wire, while the charge mode is only present at the edges. (b) A strong, nonthermal energy mode nonequilibrium $f_{L}$ suppresses superconductivity at the center of the wire.

In performing these numerical calculations, we assumed that the reservoirs are fully normal down to the lowest temperatures. The dashed lines in Fig. 2(a), however, show a schematic picture of a situation where the reservoirs are proximitized by the wire, which in fact is a situation we encounter in the experiments. The conversion and voltage drop occur primarily in the contact pads, and the measured resistance is largely a spreading resistance of the contact pad. We will show experimentally that the latter contribution can be quenched by the bias and by a magnetic field.

\section{B. Bimodal superconducting state}

A second solution was inspired by our experimental results. It consists of two separate superconducting domains located at each end of the wire [Fig. 2(b)]. A strong energy mode $f_{L}$ suppresses superconductivity in the middle of the wire, while the presence of the cold reservoirs near the ends of the wire favors locally the emergence of a gap. Modeling this state is complicated, as the presence of two superconducting regions potentially gives rise to time-dependent processes. We can, however, avoid this complication by assuming that the center of the wire is fully normal. In that case, it is possible to proceed numerically by splitting the wire in two half-wires and treat them independently, using $\theta=\nabla \chi=0$ as boundary conditions. While the distributions at the end of the wire are again given by the equilibrium reservoirs, in the middle of the wire we match the distribution functions $f_{L}$ and $f_{T}$ and their derivatives. The occupation of electronic states with energies $E+e V, E-e V$ are coupled by the applied voltage, while previously they were independent. In addition, the superconducting potential mixes particle and hole states, and one retrieves relatively complex solutions for $f_{L, T}(E, x)$ [Fig. 3(b)]. At the center of the wire, the energy mode nonequilibrium is close to a thermal one, but at an elevated temperature similar to a parabolic temperature profile. The remaining structure is in essence due to energy-conserving Andreev reflection processes, similar to the electron distribution in a superconductor - normal metal - superconductor structure. ${ }^{23}$

The emerging superconducting blobs at the end of the wire are relatively small, both in magnitude $|\Delta| \approx\left|\Delta_{0}\right| / 2$ and in size $L_{S} \approx 4 \xi$. Due to their limited size, only a tiny fraction of the total current is converted into a supercurrent, and the voltage profile is almost identical to the normal state. While the local microscopic properties at the end of the wire show a strong superconducting signature, the global properties of the wire are hardly influenced. This is true for the current (which is almost completely normal) and the voltage profile, but also for the density of states and the distribution functions. Apart from some small modifications, the distribution function in the wire is given by a two-step function. The nonequilibrium energy mode $f_{L}$ is the strongest in the center of the wire, and is the main reason why the superconducting state nucleates near the equilibrium reservoirs. The influence of $f_{T}$ is limited as the condensate carries almost no (super)current.

\section{SAMPLE DESIGN, FABRICATION, AND CHARACTERIZATION}

Figure 1(a) shows a typical superconducting Al nanowire contacted by two massive normal reservoirs, consisting of the same thin Al layer covered by a thick $\mathrm{Cu}$ layer. For reasonably clean interfaces, the inverse proximity effect of the thick $\mathrm{Cu}$ drives the Al normal down to the lowest temperatures. The massive volume of the contacts guarantees that they act as equilibrium reservoirs from which electrons are injected into the wire. When a bias is applied, however, the temperature of the reservoirs electron distribution function $f_{0}(E, T)$ might for increasing voltage deviate from the bath temperature according to $^{24}$

$$
\begin{gathered}
T^{2}=T_{0}^{2}+b^{2} V^{2}, \\
b^{2}=\frac{1}{\pi L} \frac{R_{\square}}{R_{\text {wire }}} \ln \left(\frac{r_{0}}{r_{1}}\right),
\end{gathered}
$$

where $L$ is the Lorenz number, $R_{\square}$ the sheet resistance of the contact, and $r_{0}$ and $r_{1}$, respectively, the electron-electron and electron-phonon inelastic mean-free path. The temperature increase can be considerable, and the most obvious way of decreasing it is to minimize the ratio $R_{\square} / R_{\text {wire }}$ by using thick reservoirs, which we have implemented in our sample design.

The samples are realized by three-angle shadow evaporation through a suspended resist mask (PMMA/LOR double layer), in a system with a base pressure of $0.5-1.5 \times$ $10^{-7}$ mbar. The parameters of the different samples are summarized in Table I. First, 50-90 nm of $99.999 \%$ purity $\mathrm{Al}$ is deposited through a slit in the suspended mask to create the superconducting wire and the thin bottom layer of the pads. Evaporation of a thick (200-500 nm) copper layers under an angle, which avoids deposition through the slit, completes the normal bilayers forming the reservoirs. The time between the two steps is kept to a minimum $(<10 \mathrm{~min})$ to ensure a clean and transparent interface. Subsequently, the $\mathrm{Al}$ is oxidized during $5 \mathrm{~min}$ in a pure $\mathrm{O}_{2}$ atmosphere with a pressure of $4.6 \mathrm{mbar}$ to create an $\mathrm{AlO}_{x}$ tunnel barrier of $R_{n} A \approx 300 \Omega \mu \mathrm{m}^{2}$. The $\mathrm{Cu}$ 
TABLE I. Overview of the properties of the different samples: $L$, length; $w$, width; $t$, thickness; $R_{n}$, normal-state resistance; $\rho$, resistivity; $D$, diffusion constant; $\xi=\sqrt{\hbar D / 2 \Delta}$, coherence length; $T_{c}$, critical temperature; $R_{s}$, low-temperature resistance in the superconducting state. For samples indicated with an asterisk, there is no measurement available for $T_{c}$. We assumed the same value for $T_{c}$ as for sample 3 , which was fabricated under the same conditions.

\begin{tabular}{lccccccccc}
\hline \hline$\#$ & $L(\mu \mathrm{m})$ & $w(\mathrm{~nm})$ & $t(\mathrm{~nm})$ & $R_{n}(\Omega)$ & $\rho(\mu \Omega \mathrm{cm})$ & $D\left(\mathrm{~cm}^{2} \mathrm{~s}^{-1}\right)$ & $T_{c}(\mathrm{~K})$ & $\xi(\mathrm{nm})$ & $R_{s}(\Omega)$ \\
\hline $1 \mathrm{a}$ & 1.4 & 100 & 90 & 2.8 & 1.8 & 98 & 1.23 & 131 \\
$1 \mathrm{~b}$ & 2.0 & 100 & 90 & 4.5 & 2.0 & 87 & 1.23 & 124 \\
2 & 3.0 & 200 & 50 & 3.7 & 1.23 & 143 & $1.35^{*}$ & 152 & 0.81 \\
$3 \mathrm{a}$ & 2.0 & 100 & 50 & 6.2 & 1.54 & 115 & 1.35 & 135 \\
3b & 4.0 & 100 & 50 & 13.3 & 1.66 & 106 & 1.35 & 131 \\
4 & 1.5 & 100 & 50 & 5.1 & 1.70 & 104 & $1.35^{*}$ & 129 & 1.7 \\
5 & 2.0 & 100 & 50 & 4.8 & 1.20 & 147 & $1.35^{*}$ & 154 \\
\hline \hline
\end{tabular}

probes are deposited during the last evaporation step under a second angle. The size of the wires is measured using scanning electron microscopy. The thickness was obtained from a quartz crystal monitor used during the deposition of the Al film, and calibrated by atomic force microscopy.

\section{A. Linear response of the nanowire}

Figure 6(a) shows a typical current voltage curve (IV). The linear regime extends up to a critical current designated by $I_{c 1}$. This initial slope has been measured as a function of temperature with an ac technique leading to the results shown in Fig. 4. We used a bias current $I_{12}$ of $1 \mu \mathrm{A}$ modulated at $342 \mathrm{~Hz}$ [terminal labels are shown in Fig. 1(b)]. The two-point resistance of this 1.4- $\mu \mathrm{m}$-long wire (sample 1a) as a function of temperature displays a well-defined pattern (open squares). The spreading resistance of the contact pads adds a small but finite contribution of approximately $20 \mathrm{~m} \Omega$ to the measured two-point resistance. Clearly, at high temperatures, the wire is normal and has a resistance $R_{n}$. When the temperature is decreased below $T_{c}=1.05 \mathrm{~K}$, the resistance of the wire drops considerably as it becomes superconducting. This critical temperature is depressed compared to the intrinsic critical temperature of the aluminum due to the proximity effect, as discussed by Boogaard et al. ${ }^{9}$ For intermediate temperatures (500$800 \mathrm{mK}$ ), the resistance appears to saturate at a value $R s \approx$ $1 \Omega$. As we will analyze further, this is the result of a normal current penetrating into the wire over roughly one coherence length $\xi$, yielding a $R_{S} \approx 2 \rho \xi / A$, with $A$ the cross section.

Further lowering of the temperatures leads to a further drop in resistance to almost zero, suggesting that the bilayer contacts are becoming superconducting due to a low transparency of the interface between the $\mathrm{Al}$ and $\mathrm{Cu}$ layers. To check this hypothesis, we measured the resistance of identical $\mathrm{Al} / \mathrm{Cu}$ bilayer strips down to the lowest temperatures, and find that they stay normal. Instead, we attribute the vanishing resistance due to the proximity effect by the nanowire on the contact point between the normal reservoir and the superconducting wire itself. The superconductivity gradually spreads out into the bilayer, leading to a normal-superconducting boundary which moves from the nanowire into the contact pads [Fig. 2(a)]. Consequently, the current conversion resistance in the wire itself becomes gradually less relevant. As the cross section for the conversion moves into the contacts, it becomes larger, reducing its resistive contribution. Hence, only a part of the spreading resistance $(\approx 20 \mathrm{~m} \Omega)$ is measured.

This observed pattern changes if we measure the resistance for a small dc bias current, larger than $I_{c 1}$. Then, superconductivity in the weakly proximitized region in the pads is suppressed, and the differential resistance stays constant down to the lowest temperatures [blue triangles, Fig. 4(a)]. To further test this hypothesis, we measured the $I V$ of the wire while we apply a small magnetic field of $7 \mathrm{mT}$, parallel to the pads but perpendicular to the wire [Fig. 4(b)]. The vanishing resistance at zero bias is no longer observed, while the differential resistance at higher biases is identical to the
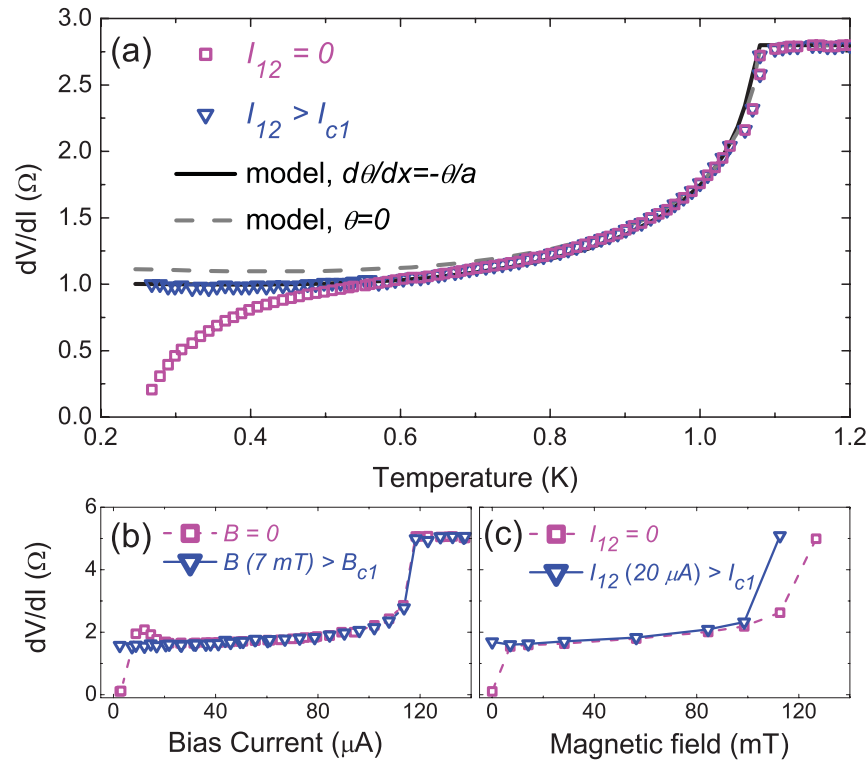

FIG. 4. (Color online) (a) The two-probe resistance versus temperature of a $1.4-\mu \mathrm{m}$-long wire (sample 1a). Due to the proximity effect of the wire on the normal reservoirs, the resistance becomes negligible at low temperatures. This weak proximity effect can be suppressed by applying a small bias current (b) or small magnetic field (c) (sample 4, $200 \mathrm{mK}$ ). This "corrected" wire resistance is constant down to the lowest temperatures [magenta squares of panel (a)]. A model (dashed line) with rigid normal boundary conditions for the pairing angle $\theta=0$ slightly overestimates the observations. A weaker boundary condition (full line), in which $\theta$ decays gradually to zero over a characteristic length $a$, shows excellent agreement with the experiment. 
one without magnetic field. This indicates that such a small field does not influence the properties of the wire, and only quenches the weakly proximitized region in the pads. Only at a much higher field $B \approx 100 \mathrm{mT}$ do we observe a change in the differential resistance of the wire [Fig. 4(c)].

The dashed gray line in Fig. 4 shows the calculated twopoint resistance. The bulk critical temperature $T_{c 0}=1.23 \mathrm{~K}$ was the only free parameter in the fit, while the diffusion constant $D=98 \mathrm{~cm}^{2} / \mathrm{s}$ was obtained through the relation $D=\rho / N_{0} e^{2}$. The resistivity $\rho$ is deduced from the normalstate resistance $R_{n}$, using $N_{0}=2.2 \times 10^{47} \mathrm{~J}^{-1} \mathrm{~m}^{-3}$ for the density of states at the Fermi level. ${ }^{25}$ The superconducting coherence length is obtained from $\xi=\sqrt{\frac{\hbar D}{2 \Delta}}$. Although the numerical calculation agrees quite well with the data (for $I>I_{c 1}$ ), the model overestimates the residual resistance at low temperatures. This indicates that the assumption of completely normal contact pads is too rigid, as also observed by Boogaard et $a l .{ }^{9}$ To include the geometric out-diffusion of coherent electrons into the normal pads, we adjust the boundary conditions at the ends of the wire to $\nabla \theta=-\theta / a$, which indicates the dilution of superconductivity into the normal pads over a characteristic length scale $a$. With $a \approx 18 \mathrm{~nm}$ (full line in Fig. 4), we find excellent agreement with the observations. The key parameters are listed in Table I for the different samples. It demonstrates that the linear response of the wires is well understood, but that the boundary conditions are a sensitive part of the problem even for the thick and wide contact pads used. However, for bias currents $I \geqslant I_{c 1}$, the system is in a well-defined state, which can be connected to the theoretical predictions.

\section{B. Characterization of the tunnel probe}

To measure locally the density of states, the electrostatic potential $e \phi(x)$, and the chemical potential of the condensate $\mu_{c p}$, we use a normal tunneling probe. The current flowing from a normal tunnel probe contacted to a nonequilibrium superconductor at a position $x$ is given by

$$
\begin{aligned}
I_{T}(V, x)= & \frac{1}{e R_{n}} \int_{-\infty}^{\infty} \operatorname{Re}\{\cos [\theta(E, x)]\} \\
& \times\left\{f_{T}^{S}(E, x)-f_{T}^{N}(E+e V)\right\} d E,
\end{aligned}
$$

with $\theta(E, x)$ the pairing angle, $f_{T}^{S}(E, x)$ the charge mode nonequilibrium distribution in the superconductor, and $f_{T}^{N}=$ $1-f_{0}(E+e V)-f_{0}(-E+e V)$ the distribution function in the normal probe. Using Eq. (5), we can rewrite this to

$$
I_{T}(x)=\frac{1}{e R_{n}}\left\{e \phi(x)-\int_{-\infty}^{\infty} N(E, x) f_{T}^{N} d E\right\} .
$$

The tunnel current consists of two contributions: the first one does not depend on the applied voltage, but is completely determined by the charge imbalance in the superconductor, leading to the local electrostatic potential $\phi(x)$. The second contribution is given by the convolution of the local DOS $N(E, x)$ of the superconductor and the distribution function $f_{T}^{N}(E+e V)$ in the normal metal. At low temperatures, the differential conductance of the tunnel contact is a direct measure for the density of states in the superconductor. The

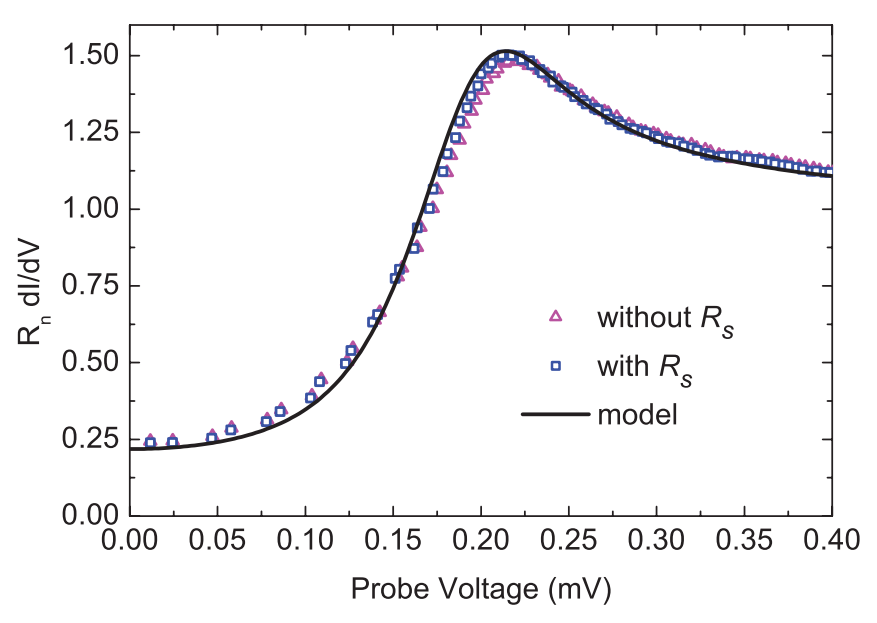

FIG. 5. (Color online) The differential conductance of the local tunnel probe as a function of applied voltage (magenta triangles). Good agreement between experiment and theory (full line) is obtained when the series resistance of the setup is included (blue squares).

condensate chemical potential of the superconductor can be obtained from $N(E, x)$, which is symmetric around $E=\mu_{c p}$.

Figure 5 shows a typical measurement of the differential resistance for a tunnel probe located at a distance of $320 \mathrm{~nm}$ $=2.4 \xi$ from the normal reservoir of a $4-\mu \mathrm{m}$-long wire. The nanowire is biased just above $I_{c 1}$ to ensure it is in a well-defined state. The bias current needed to drive the probe is typically four orders of magnitude smaller than the bias current of the nanowire, due to the high normal resistance of the tunnel junction $\left(R_{T}=43 \mathrm{k} \Omega\right)$. Hence, it is safe to assume that the properties of the nanowire are not influenced by the measurement of the probe. One recognizes the coherence peaks at the gap voltage, however, the subgap DOS is increased in comparison to the BCS values due to the presence of the normal banks, and the driving of the nanowire. The simulated local DOS, for the set of parameters, is in good agreement with the data, but near the gap voltage a small discrepancy exists. We attribute this to a series resistance in the wiring of the tunnel probe and can correct our data for this contribution. We obtain a good agreement between the data and the theory using a series resistance of $R_{S}=1.2 \mathrm{k} \Omega$, which is the estimated wiring resistance of the experimental setup.

\section{TWO-STATE ANALYSIS AND DISCUSSION}

We have realized and studied a total of seven samples with parameters shown in Table I. All displayed similar behavior. The nonlinear current voltage characteristic of a typical sample is shown in Fig. 6(a), with two clearly distinguished branches. Before discussing the details, we first indicate the various signatures for processes, which dominate the various regimes. By increasing the current from zero bias, we pass $I_{c 1}$, the current at which the proximitization in the banks is quenched as discussed in Sec. IV A. Beyond $I_{c 1}$ until $I_{c 2}$, we claim that the wire remains in the global superconducting state, characterized by a low and almost constant differential resistance $R_{s}$ [Fig. 6(b)]. This resistance reflects the conversion of a normal current into a supercurrent and is located at the edges of the wire. At the current $I=I_{c 2}$, the wire switches 

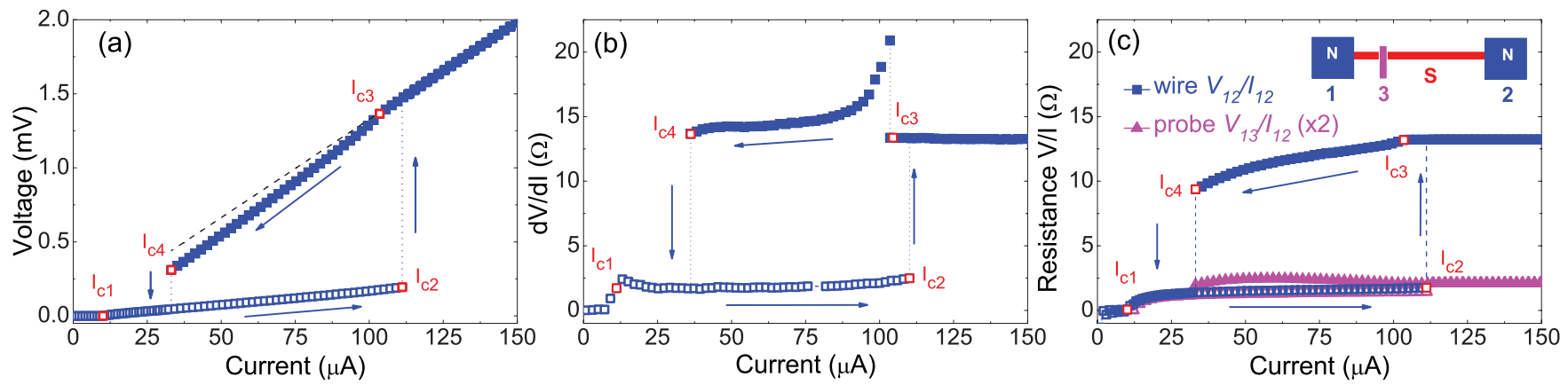

FIG. 6. (Color online) The voltage $V_{12}$ (a) and differential resistance (b) of a 4- $\mu$ m-long wire (sample $3 \mathrm{~b}$ ) as a function of bias current $I_{12}$, measured at $200 \mathrm{mK}$. We define four different regimes with boundaries labeled $I_{c 1}-I_{c 4}$, each characterized by a nearly constant differential resistance. The critical currents $I_{c 2}$ and $I_{c 4}$ are defined as the bias currents where the wire switches between the two hysteretic voltage branches. $I_{c 1}$ and $I_{c 3}$ are the transition points between the two different states of one branch. (c) The apparent resistance of the complete wire $V_{12} / I_{12}$, and of the edge of the wire $V_{13} / I_{12}$ as measured with a voltage probe, multiplied by two for the ease of comparison.

into the normal state, leading to an abrupt switch of both the voltage and the differential resistance, followed by a constant differential resistance equal to the normal-state resistance.

Decreasing the current from the normal state, a kink in the measured voltage signals a more subtle transition at $I=I_{c 3}$. The measured voltage shows a small deficit with respect to its normal-state value (black dashed line), suggesting the nucleation of superconductivity. We claim that superconductivity nucleates here at the ends of the wires close to the contact pads in agreement with Fig. 2(b). The sudden transition at $I=I_{c 4}$ is due to the transition from the bimodal to the global superconducting state.

A first experimental indication to support this interpretation is provided by Fig. 6(c), which compares local measurements with measurements over the full wire. It shows the two-point resistance of the wire $V_{12} / I_{12}$ (squares) as a function of bias current $I_{12}$, together with the apparent resistance $V_{13} / I_{12}$ (triangles) at the ends of the wire (see inset for the probe position and terminal labels). The probe voltage is multiplied by two for comparison, as a similar contribution is present at the other edge of the wire. For the lower branch, the assumed global superconducting state, one observes that the voltage drop $V_{13}$ over the end of the wire is almost identical to half of the complete voltage drop over the wire length, a direct proof that this resistance is located at the ends of the wire.

In contrast, in the normal state, the voltages $V_{12}$ and $V_{13}$ are, as expected, proportional to their respective lengths along the wire, $V=\rho L / A$. Upon decreasing the bias below $I_{c 3}$, where we assume the bimodal state exists, one observes over the full length of the wire a decreasing resistance for decreasing bias, signaling the growing strength of superconductivity somewhere. The measured resistance over the end of the wire, however, increases compared to the normal state $V_{13} / I_{12} \geqslant R_{n}$. Although counterintuitive, this is consistent with the general nonequilibrium present in the superconductor. In the following, we make a detailed analysis of both superconducting states, and place the experimental results in the context of the theoretical model.

\section{A. Global superconducting state}

Figure 7 shows two-point measurements of the lower branch of a 1.4- $\mu$ m-long nanowire (sample 1a) at three different bath temperatures. In view of the analysis shown Sec. IV A, we assume that the resistance of the wire is primarily determined by the charge mode of the distribution function $f_{T}(E, x)$, which depends on the position-dependent density of states and the order parameter $\Delta(x)$. The weak dependence of the differential resistance on the current indicates that the superconducting properties of the wire hardly change with increasing bias (open symbols). Although numerical simulations (filled symbols in Fig. 7) show the same qualitative behavior, the simulations seem to overestimate the bias current at which the differential resistance begins to increase. Hence, the observed switching current $I_{c 2}$ is also slightly lower than predicted. Ignoring this small discrepancy, the simulated data show good agreement with the experiment over the complete

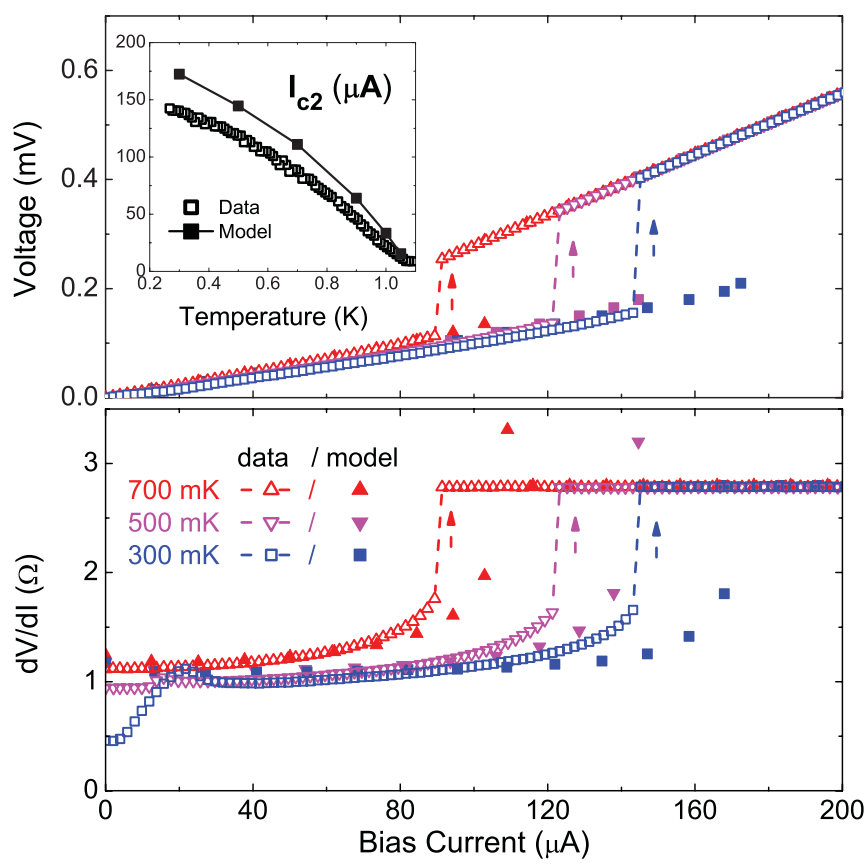

FIG. 7. (Color online) Two-probe voltage (a) and differential resistance (b) as a function of bias current, for a 1.4- $\mu \mathrm{m}$ wire, at three different bath temperatures. Open symbols: experimental data. Filled symbols: numerical simulations. The critical current as a function of temperature (inset). 


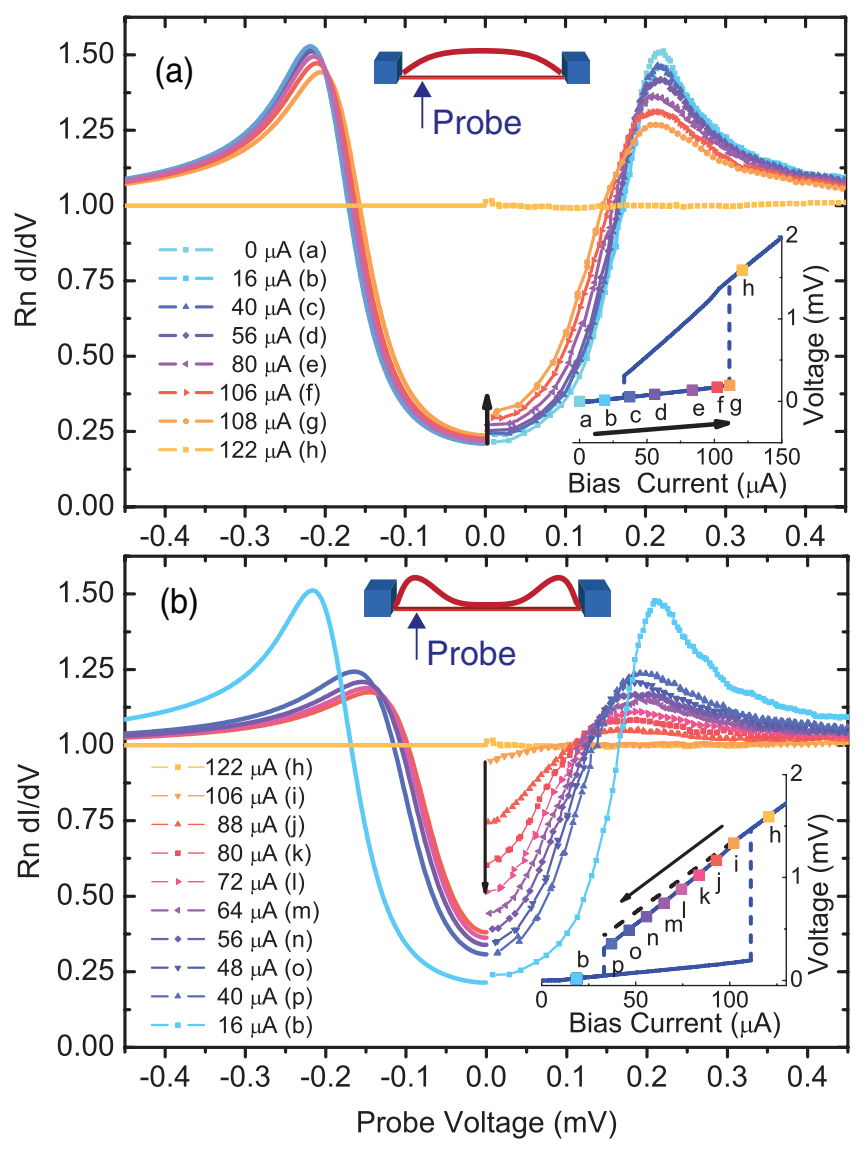

FIG. 8. (Color online) The local density of states for (a) the global superconducting state and (b) the bimodal state, for different bias currents $I_{12}$ of the nanowire, measured at $200 \mathrm{mK}$. For the global superconducting state, the gap is only weakly dependent on the bias current, while for the bimodal state, one observes a DOS gradually changing from a normal into a superconducting state.

temperature range (inset Fig. 7). At the same time, the observed values for the critical current (or critical voltage) are much smaller than what one would expect for a pair-breaking current, experimentally ${ }^{26,27}$ as well as theoretically. ${ }^{28,29}$ This demonstrates that the nonequilibrium processes should be taken into account in evaluating the parameters. The remaining deviations between theory and experiment suggest, most likely, that the temperature of the reservoirs deviates from the bath temperature for higher driving currents, as expected from Eq. (8).

Figure 8 (a) shows the differential conductance $d I_{13} / d V_{13}$ of a tunnel probe, located at a distance of $320 \mathrm{~nm}(=2.4 \xi)$ from the normal reservoir of a $4-\mu \mathrm{m}$-long wire (sample $3 \mathrm{~b}$ ). The wire is biased at a fixed current $I_{12}$ with a corresponding voltage $V_{12}$. At the same time, the probe current $I_{13}$ is varied while measuring the probe voltage $V_{13}$ [Fig. 1(b)]. The evolution of the local DOS for increasing bias is shown on the right side in Fig. 8(a). The conductance at zero bias $V_{p}=0$ increases slightly for increasing bias, while the coherence peaks get further smeared out. The dependencies are, however, weak, and even right before the switching current $I_{c 2}$ the DOS is hardly affected by the drive current. These observations are in close agreement with the theoretical predictions and confirm the idea that the superconducting state remains globally stable. For increasing bias, the resistance remains located at the ends of the wire and the DOS does not change either. Unfortunately, we have not been able to directly measure $f_{L}(x)$. Nevertheless, we believe that this energy mode nonequilibrium triggers the transition at $I_{c 2}$ as analyzed by Keizer et al. ${ }^{7}$

\section{B. Bimodal superconducting state}

The continuous transition, with decreasing bias, from the normal into a superconducting state at $I_{c 3}$ (Fig. 6) indicates that the emerging superconducting state is initially very close to the normal state. For lower bias currents, the absolute resistance gradually decreases [Fig. 6(c)], which suggests that an increasing fraction of the current is carried by the emerging condensate [Fig. 2(b)]. A similar picture is observed for the local density of states, plotted in Fig. 8(b) for different bias currents $I_{12}$ of the nanowire. Below $I_{c 3}$, a gradually increasing gap is found, unambiguously showing the emergence of superconducting order. Close to $I_{c 3}$, the DOS at the position of the probe evolves in a continuous way from a flat spectrum into a spectrum with a gap. However, at $I_{c 4}$ one observes an abrupt transition to a situation with a stronger gap. The abruptness indicates that it is a transition from two distinct superconducting states, which directly proves that at least two microscopically distinct superconducting states exist. Although the simulations for the local DOS agree well at currents close to $I_{c 4}$, they do not account in detail for the gradual evolution between the normal and superconducting states at $I_{c 3}$. At this point, we assume that the reservoirs start to heat up, and can no longer be treated as equilibrium reservoirs with $T=T_{0}$. Overall, the model supports the picture of the emergence of the superconducting state quite nicely, with the strongest nonequilibrium in the wire occurring at $I_{c 4}$, with the reservoirs most closely to equilibrium at $T=T_{0}$.

The electrochemical potential of the superconducting condensate $\mu_{c p}$ is determined from the minimum of the measured DOS $\mu_{c p}=\left.e V\right|_{\min (\mathrm{DOS})}$. It is found that, at this probe position, $\mu_{c p}$ is equal to the electrostatic potential $V_{1}$ of the adjacent reservoir. Measurements with a probe in the middle of the wire show that, in the same bias regime, the voltage is equal for both sides of the wire, which means that the state is symmetric, and a similar superconducting region should exist near the other reservoir at a potential $V_{2}$. If these two regions were part of one global superconducting state, there would be a voltage drop $\Delta V=V_{1}-V_{2}$ over the superconducting potential $\mu_{c p}$ of this state, and a superconducting phase-slip process should occur. However, according to the Josephson relation $2 e V=\partial \chi / \partial t$, it would be at a frequency $v \approx 8 \hbar / \Delta$, which is too high compared to the energy gap.

The fact that the two-point resistance is so close to $R_{n}$, the gradual increase of the DOS at the position of the probe, and the electrochemical potential of the condensate demonstrate that two separate superconducting regions emerge at the edges of the wire. The physical reason is the energy mode nonequilibrium, as discussed by Keizer et al. ${ }^{7}$ for the lower branch, but similarly for this upper branch. At the bias $I_{c 4}$, the wire is still largely normal and $f_{E}$ is given by the two-step distribution function. In the middle of the wire, the width of the step is several times bigger than the superconducting gap. 
Through relation Eq. (6), it is seen that this suppresses fully the nucleation of a gap, while the cold equilibrium reservoirs favor the emergence of a gap at the edges of the wire. Simply put, the ends are cold where the center of the wire is hot. Therefore, we conclude that the results are most easily understood as due to two distinct superconducting domains, separated by a normal central region, which we have called the bimodal state.

Finally, we discuss the voltage $V_{13}$ measured by the probe when the wire is biased into the bimodal state [triangles in Fig. 6(c)]. Close to $T_{c}$, the voltage measured by such a normal probe is equal to the electrochemical potential of the quasiparticle bath. ${ }^{17}$ At low temperatures ${ }^{16}$ and for short-lived quasiparticles, ${ }^{18}$ it is impossible to define a quasiparticle bath with a well-defined chemical potential; however, the measured voltage is still related to the local electrostatic potential $e \phi$ [using Eq. (10)]:

$$
e \phi=\int_{-\infty}^{\infty} N(E) f_{T}^{N}(E+e V) d E .
$$

For a relatively small charge imbalance $e \phi(x) \ll \Delta$, the measured voltage equals the local electrostatic potential $e \phi(x)$ divided by the local DOS in the superconductor at zero energy: $V \approx \phi / N(0)$. Hence, the voltage measured with the tunnel probe can be larger than the local potential $e \phi(x)$.

\section{CONCLUSION}

We have analyzed a well-defined model system of a superconducting wire between two massive normal contact pads. We demonstrate that this system, when driven by a current, has two distinct metastable superconducting states. For low bias, we find a global superconducting state with most of the resistance occurring as a current-conversion resistance at the ends of the superconducting wires where normal current enters. Although resistive, we demonstrate that the whole wire including the edges continues to be in one coherent superconducting state. This state does hardly change for increasing current until the wire switches abruptly to the full normal state at a current, which is much lower than the critical pair-breaking current. On a microscopic level, the distribution function changes considerably and is strongly different from the commonly used parabolic temperature profile. A numerical analysis based on the nonequilibrium quasiclassical Green's functions shows that the switching current is determined by the nonequilibrium electron distributions, in good agreement with the experimental results.

For high bias, decreasing the current from a fully normal state, we find that the superconducting state emerges as two decoupled domains at the ends of the wire. The vicinity of the cool equilibrium reservoirs favors the nucleation of the superconducting state at these ends, while strong nonequilibrium at the center of the wire continues to suppress the superconductivity. Upon further lowering of the bias current, the two domains grow in strength until the wire switches back to the low resistive, globally superconducting state. We speculate that the transition from one state to the other is triggered by a condition in which the Josephson coupling energy between the two domains exceeds the thermal energy at that bias point.

This work is also relevant for normal-metalsuperconductor-normal-metal mixing devices, called hot-electron bolometer (HEB) mixers. ${ }^{30}$ In most practical cases, the superconducting material is thin $\mathrm{NbN}$ and gold $(\mathrm{Au})$ normal pads are used as antenna. Under the condition that no radiation is applied to an HEB, the present analysis is helpful to understand the observed current-voltage characteristics, which are analogous to the one shown in Fig. 6(a). ${ }^{31}$ The resistive properties for low bias and temperature will be dominated by the conversion resistance at the interfaces (controlled by $f_{T}$ ). This regime will extend to a critical current, analogous to $I_{c 2}$ reported here, but with a value which may depend on the electron-phonon relaxation, which is present in a material like $\mathrm{NbN}$, but is negligible in our experiment with $\mathrm{Al}$. Beyond this critical value, the device is most likely either fully in the normal state (beyond $I_{c 3}$ as identified here), or in the bimodal state (for lower biases between $I_{c 3}$ and $I_{c 4}$ ). The stronger electron-electron and electron-phonon interaction in $\mathrm{NbN}$ as compared to Al will bring the longitudinal nonequilibrium $f_{L}$ closer to a local thermal profile. In case radiation is applied to an HEB, an overall increase in electron temperature occurs, which brings the superconductor close to its transition point where thermally activated phase-slip events contribute to the resistivity. Hence, for a full understanding of the HEB mixers, one needs to take into account two contributions to the observed resistance: first the static conversion resistance inside the superconductor near the interface between the normal metal and the superconductor, described here, dominating for the unexposed devices, and second the resistance due to time-dependent phase-slip events occurring at electron temperatures close to the critical temperature of the superconductor, which dominates under actual mixer operation. ${ }^{4}$

\section{ACKNOWLEDGMENT}

We would like to acknowledge Nanofridge (Nanosci-ERA), Microkelvin (No. 228464, Capacities Specific Programme), and the Foundation for fundamental research on matter (FOM).
*Current address: University of Leiden, Leiden, The Netherlands.

${ }^{1}$ A. V. Gurevich and R. G. Mints, Rev. Mod. Phys. 59, 941 (1987).

${ }^{2}$ M. Tinkham, J. U. Free, C. N. Lau, and N. Markovic, Phys. Rev. B 68, 134515 (2003).

${ }^{3}$ N. Shah, D. Pekker, and P. M. Goldbart, Phys. Rev. Lett. 101, 207001 (2008).
${ }^{4}$ R. Barends, M. Hajenius, J. R. Gao, and T. M. Klapwijk, Appl. Phys. Lett. 87, 263506 (2005).

${ }^{5}$ H. Pothier, S. Guéron, N. O. Birge, D. Esteve, and M. H. Devoret, Phys. Rev. Lett. 79, 3490 (1997).

${ }^{6}$ A. Schmid and G. Schön, J. Low Temp. Phys. 20, 207 (1975). 
${ }^{7}$ R. S. Keizer, M. G. Flokstra, J. Aarts, and T. M. Klapwijk, Phys. Rev. Lett. 96, 147002 (2006).

${ }^{8}$ A. Sanchez-Canizares and F. Sols, J. Low Temp. Phys. 122, 11 (2001).

${ }^{9}$ G. R. Boogaard, A. H. Verbruggen, W. Belzig, and T. M. Klapwijk, Phys. Rev. B 69, 220503 (2004).

${ }^{10}$ W. J. Skocpol, M. R. Beasley, and M. Tinkham, J. Appl. Phys. 45, 4054 (1974).

${ }^{11}$ A. F. Volkov and S. M. Kogan, JETP Lett. 19, 4 (1974) [Pis'ma Zh. E'ksp. Teor. Fiz. 19, 9 (1974)].

${ }^{12}$ N. Kopnin, Theory of Nonequilibrium Superconductivity, International Series of Monographs on Physics, Vol. 110 (Oxford University Press, New York, 2001).

${ }^{13}$ K. D. Usadel, Phys. Rev. Lett. 25, 507 (1970).

${ }^{14}$ I. Snyman and Y. V. Nazarov, Phys. Rev. B 79, 014510 (2009).

${ }^{15}$ T. H. Stoof and Y. V. Nazarov, Phys. Rev. B 53, 14496 (1996).

${ }^{16}$ J. Clarke, Phys. Rev. Lett. 28, 1363 (1972).

${ }^{17}$ C. J. Pethick and H. Smith, Ann. Phys. (NY) 119, 133 (1979).

${ }^{18}$ J. B. Nielsen, C. J. Pethick, J. Rammer, and H. Smith, J. Low Temp. Phys. 46, 565 (1982).

${ }^{19}$ F. Hübler, J. C. Lemyre, D. Beckmann, and H. v. Löhneysen, Phys. Rev. B 81, 184524 (2010).
${ }^{20}$ R. Yagi, Phys. Rev. B 73, 134507 (2006).

${ }^{21}$ P. Santhanam and D. E. Prober, Phys. Rev. B 29, 3733 (1984).

${ }^{22}$ S. B. Kaplan, C. C. Chi, D. N. Langenberg, J. J. Chang, S. Jafarey, and D. J. Scalapino, Phys. Rev. B 14, 4854 (1976).

${ }^{23}$ F. Pierre, A. Anthore, H. Pothier, C. Urbina, and D. Esteve, Phys. Rev. Lett. 86, 1078 (2001).

${ }^{24}$ M. Henny, S. Oberholzer, C. Strunk, and C. Schönenberger, Phys. Rev. B 59, 2871 (1999).

${ }^{25}$ N. W. Ashcroft and N. D. Mermin, Solid State Physics (Saunders College, New York, 1976).

${ }^{26}$ J. Romijn, T. M. Klapwijk, M. J. Renne, and J. E. Mooij, Phys. Rev. B 26, 3648 (1982).

${ }^{27}$ A. Anthore, H. Pothier, and D. Esteve, Phys. Rev. Lett. 90, 127001 (2003).

${ }^{28}$ M. Yu. Kupriyanov and V. F. Lukichev, Sov. J. Low Temp. Phys. 6, 210 (1980) [Fiz. Nizk. Temp. 6, 445 (1980)].

${ }^{29}$ J. Bardeen, Rev. Mod. Phys. 34, 667 (1962).

${ }^{30}$ D. E. Prober, Appl. Phys. Lett. 62, 2119 (1992).

${ }^{31}$ M. Hajenius, Z. Q. Yang, J. R. Gao, J. J. A. Baselmans, T. M. Klapwijk, B. Voronov, and G. Goltsman, IEEE Trans. Appl. Supercond. 17, 399 (2007). 